\title{
Using Gelatin as Protecting Agent and Organic Template to Synthesize Noble Metal Nanoparticles and Metal Nanoparticles@Mesoporous Silica for SERS and CO Oxidation Applications
}

\author{
Hsuan-Jung Chang, Ying-Mei Yang1, Chi-Chang Lin ${ }^{1}$, Yi-Chia Luo², \\ Hsien-Chang Chang², Hong-Ping Lin*, Yu-Cheng Lin', \\ Chih-Yuan Tang ${ }^{5}$ and Chin-Yen Lin ${ }^{5}$
}

\begin{abstract}
Department of Chemistry, National Cheng Kung University, Tainan, Taiwan, 701 ${ }^{1}$ Institute of Biomedical Engineering, National Cheng Kung University, Tainan, Taiwan, 701

${ }^{2}$ Department of Chemistry, National Taiwan University, Tainan, Taiwan, 106

${ }^{3}$ Center for Micro/Nano Science and Technology, Taian, Taiwan 701

${ }^{4}$ Department of Engineering Science, National Cheng Kung University, Tainan, Taiwan, 701

${ }^{5}$ Instrumentation Center, National Taiwan University, Taipei, Taiwan 106
\end{abstract}

(Received July 31, 2008; accepted November 13, 2008)

Key words: nanoparticles, mesoporous silica, gelatin, template, CO oxidation, SERS

With excellent control of their synthetic compositions and condition, noble metal nanoparticles@mesoporous silicas of large surface area $\left(\approx 300 \mathrm{~m}^{2} \mathrm{~g}^{-1}\right)$, large pore size $(>5.0$ $\mathrm{nm})$, and high noble metal nanoparticle content $(\approx 10 \mathrm{wt} . \%)$ have been conveniently prepared using natural polymer gelatin with many amide $\left(-\mathrm{CO}-\mathrm{NH}_{2}\right)$ groups as an organic template of mesoporous silica and a protecting agent for stabilizing Au nanoparticles. This synthetic method is extensive and can be used to prepare $\mathrm{Au}, \mathrm{Ag}$, and Ag-Au alloy nanoparticles@mesoporous silicas. Because the metal nanoparticles are accessible to the environment, noble-metal nanoparticles@mesoporous silicas can be used in SERS applications and act as superior catalysts in $\mathrm{CO}$ oxidation at high temperatures. In addition, bare Au nanoparticles anchored on a silica matrix can have direct contact with $S$. aureus cells to enhance the Raman spectrum intensity of $S$. aureus cells absorbed onto a Au nanoparticles@mesoporous silica.

\section{Introduction}

Mesoporous silicas of large surface area, large pore volume, and tunable pore size have attracted much attention in the chemical, biochemical, and material communities. ${ }^{(1)}$ Owing to their high stability and low biotoxicity, mesoporous silicas can be considered as a superior solid matrix for the incorporation of nanoparticles and catalytic reaction sites. ${ }^{(2-4)}$

${ }^{*}$ Corresponding author: e-mail: hplin@mail.ncku.edu.tw 
In typical synthetic methods for nanoparticles containing mesoporous silica, complicated synthetic procedures including the preparation of mesoporous silicas, surfactant removal, and the chemical vapor deposition of the precursor of the nanoparticles are required.

The cationic and neutral block-copolymer surfactants of amphiphilic property have been widely used as the mesostructural template. Pore size is, thus, mainly determined by hydrophobic chain length and volume. However, the hydrophobic parts of surfactants decompose slowly under ambient condition. With the recent increasing concern on the aquatic toxicity of surfactants, the use of environmentally friendly reagents to prepare mesoporous silica is very desirable.

According to silica chemistry, ${ }^{(5)}$ gelatin, a natural protein with many amine functional groups, can have a high affinity to strongly interact with silanol groups on silicate species via multiple hydrogen bonds. In addition, it is well known that gelatin with proper functional groups (i.e., $-\mathrm{NH}_{2}$; $-\mathrm{SH}$ ) can also act as the protecting agent of noble metal nanoparticles. Therefore, gelatin could act as both the organic template of porous silica and the protecting agent of metal nanoparticles. ${ }^{(6,7)}$

Accordingly, we proposed a simple one-pot synthetic method of preparing the metal NPs@mesoporous silicas. In the first step, a typical reduction synthetic procedure was applied to the preparation of a metal nanoparticle aqueous solution in the presence of gelatin. Then, adding an appropriate amount of sodium silicate solution at suitable pHs $(\approx 4.0$ 6.0), metal NPs@organic template-silica composites would be readily formed within a short reaction time. After calcination or solvent extraction, an organic template freemetal NPs@mesoporous silica should be obtained. Because of the excellent dispersion and accessibility of Au nanoparticles, Au nanoparticles@mesoporous silica demonstrates catalytic capability toward CO oxidation ${ }^{(8,9)}$ and SERS function. ${ }^{(10-12)}$

\section{Experimental Methods}

To prepare noble metal nanoparticles@mesoporous silica, we proposed a simple onepot synthetic method. A clear solution was formed by combining $50.0 \mathrm{ml}$ of $1.0 \mathrm{mM}$ salt (i.e., $\mathrm{HAuCl}_{4}, \mathrm{AgNO}_{3}$ ) aqueous solution with a mixture of $0.2 \mathrm{~g}$ of gelatin and $20.0 \mathrm{~g}$ of water at $4^{\circ} \mathrm{C}$. To this solution, $20.0 \mathrm{ml}$ of $5.3 \mathrm{mM} \mathrm{NaBH}_{4}$ solution was added dropwise. After that, a noble metal nanoparticle solution was formed. Then, the noble metal nanoparticlesgelatin solution was poured into an acidified silicate solution $(\mathrm{pH} \approx 5.0-6.0)$ at $40^{\circ} \mathrm{C}$, containing $2.0 \mathrm{~g}$ of sodium silicate and $100.0 \mathrm{~g}$ of water. The resulting gel solution was hydrothermally treated at $100^{\circ} \mathrm{C}$ for $1 \mathrm{~d}$. Filtration, drying, and calcination at $560^{\circ} \mathrm{C}$ for 6 h gave a noble metal nanoparticles@mesoporous silica.

Catalytic activity for $\mathrm{CO}$ oxidation was carried out in a U-tube reactor (i.d. $6.0 \mathrm{~mm}$ ) mounted in a constant-temperature zone of an electric furnace. A sample of $40.0 \mathrm{mg}$ was packed between two layers of glass wool. Prior to the reaction, the catalysts were treated with $10 \% \mathrm{H}_{2} / \mathrm{N}_{2}$ at $600^{\circ} \mathrm{C}$ for $1 \mathrm{~h}$. The reactant gases were purified using $4 \AA$ molecular sieves, then mixed and passed into the reactor. The catalytic activity was investigated in the temperature range of $25-240^{\circ} \mathrm{C}$ with mixed reactant gases, containing $1 \% \mathrm{CO}, 1 \%$ $\mathrm{O}_{2}$, and He balanced at a total flow rate of $66 \mathrm{ml} \mathrm{min}^{-1}\left(\mathrm{GHSV}=100,000 \mathrm{~g}_{\mathrm{cat}}^{-1} \mathrm{~h}^{-1}\right)$ under atmospheric pressure. CO conversion rate was based on the carbon dioxide formation divided by the total amount of $\mathrm{CO}$ fed to the catalyst. 


\section{Results and Discussion}

The Au or Ag nanoparticle aqueous solution was readily obtained from the reduction of the metal ion solution in the presence of gelatin as the protecting agent. Figures 1(a) and 1(b) show representative TEM images of the resulting $\mathrm{Au}$ and Ag nanoparticles. The average Au nanoparticle size is approximately $5.0 \mathrm{~nm}$, and the average Ag alloy nanoparticle size is relatively larger at approximately $10.0 \mathrm{~nm}$. The HR TEM image of the Ag nanoparticles clearly reveals the lattice fringes of the silver nanoparticles (Fig. 1(c)). To further characterize the nanoparticles, the UV-vis spectra of the Au and Ag@gelatin aqueous solution that exhibits broad absorption bands centered at 518 and $420 \mathrm{~nm}$, respectively, were observed (Fig. 1(d)). These results indicate that the gelatin can act as a capping agent to prevent the aggregation of Au nanoparticles with high surface energy. These results indicate that the simple reduction method for noble metal nanoparticles in the presence of gelatin has already been well established.
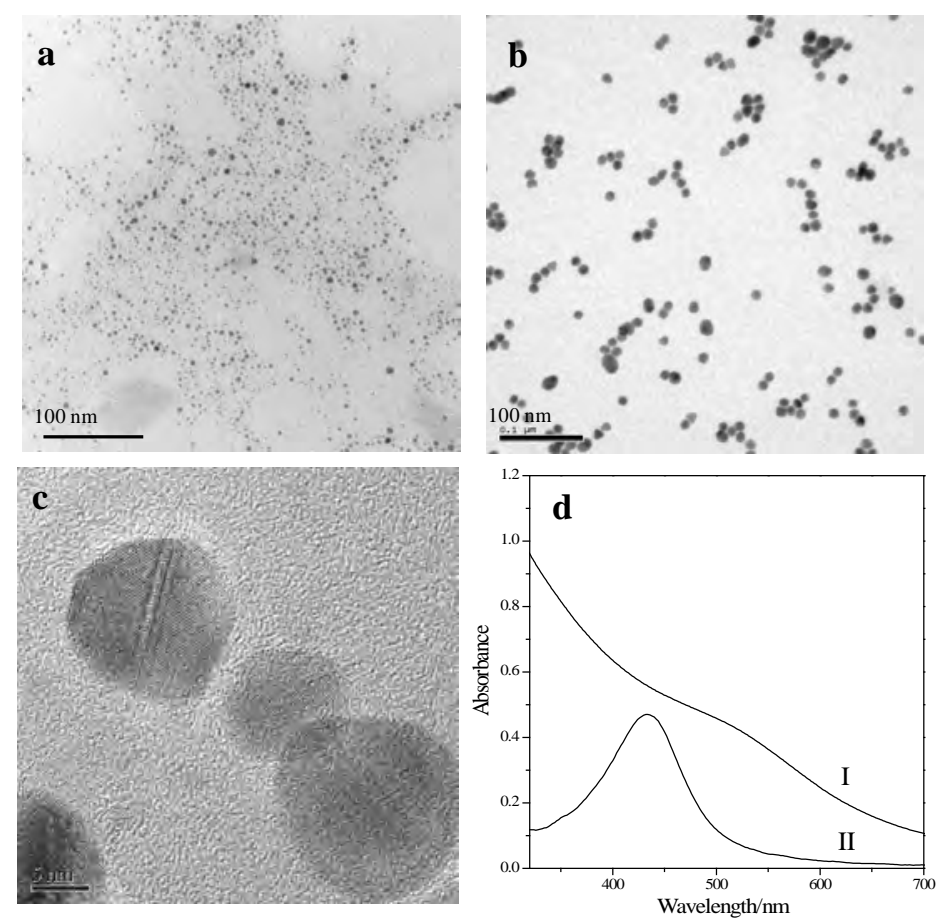

Fig. 1. TEM images and UV-vis spectra of gelatin-stabilizing noble metal nanoparticle solution at $\mathrm{pH} \approx 6.0$. (a) TEM image of Au nanoparticles; (b) TEM image of Ag nanoparticles; (c) HRTEM image of Ag nanoparticles; (d) UV-vis spectra: curve I: Au nanoparticles; curve II: Ag nanoparticles. 
Based on silica chemistry, ${ }^{(5)}$ a gelatin natural polymer with many amino groups can also assemble with silica species via hydrogen-bonding interactions at a $\mathrm{pH}$ of approximately 7 . Accordingly, after fast silicification in a dilute silicate solution at $\mathrm{pHs}$ $\approx 5.0-6.0$, the noble metal nanoparticles were completely embedded within the silica matrix within a few minutes. After filtration and calcination to remove the organic protecting agent, a red powder was generated. Figures 2(a)-2(d) show TEM images at
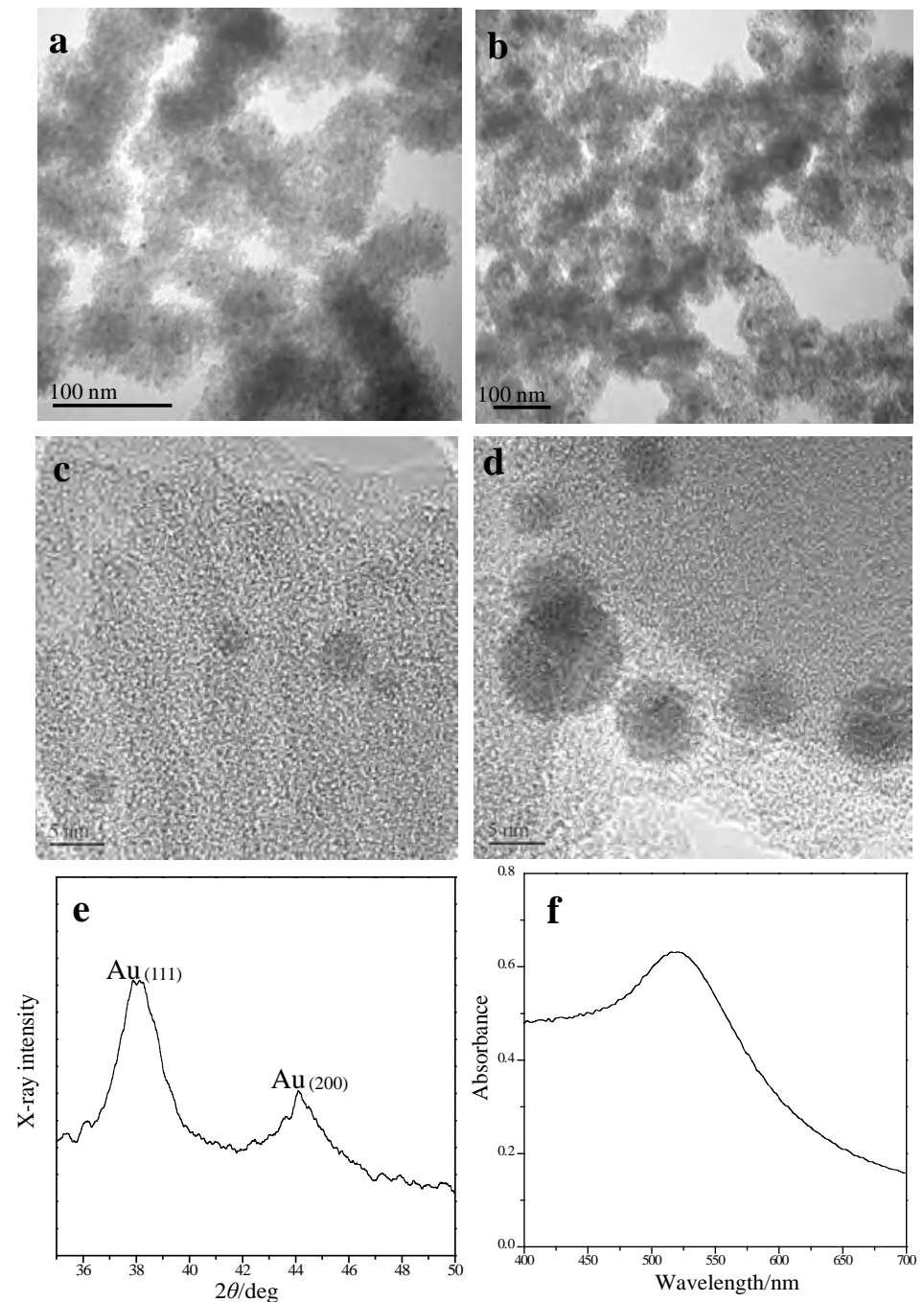

Fig. 2. (a) TEM image of Au nanoparticles@mesoporous silica; (b) TEM image of calcined Ag nanoparticles@mesoporous silica; (c) HR-TEM image of Au nanoparticles@mesoporous silica; (d) HR-TEM image of calcined Ag nanoparticles@mesoporous silica; (e) High-angle XRD pattern of Au nanoparticles@mesoporous silicas templated with gelatin; (f) Reflectance UV-vis spectrum. 
different magnifications of the Au and Ag nanoparticles@mesoporous silica samples after calcination. Owing to the confinement effect of the solid silica matrix, the noble metal nanoparticle size is still less than $10 \mathrm{~nm}$ even after hydrothermal treatment and high-temperature calcination. As in the original nanoparticle solution, the size of the Ag nanoparticles@mesoporous silica is larger than that of the Au nanoparticles. From the HR TEM images, one can clearly see lattice fringes of the Au and Ag nanoparticles (Figs. 2(c) and 2(d), respectively). Besides showing local observations by TEM, Fig. 2(e) demonstrates the high-angle XRD pattern of the Au nanoparticles@mesoporous silica after calcination. Two broad peaks at high angles of 38.4 and $44.6^{\circ}$ representing the (111) and (200) lattice planes of the Au nanoparticles, respectively, were easily found. Using the Scherrer equation (particle $\operatorname{size}=0.90 \lambda / \beta \cos \theta ; \lambda$ : wavelength of $\mathrm{X}$-ray; $\beta$ : full width at half maximum; $\theta$ : central position of diffraction peak), the average size of the Au nanoparticles after calcination was estimated to be about $7.5 \mathrm{~nm}$. This result is consistent with that of TEM. From these data, one can determine that calcination leads to an increase in the particle size of Au nanoparticles, but growth extent was particularly suppressed to only $50 \%$ by the hard mesoporous silica matrix, and hence the properties of $\mathrm{Au}$ nanoparticles were preserved. In the reflectance UV-vis spectrum of the Au nanoparticles@mesoporous silica, an absorption band centered at 520 nm further characterized the Au nanoparticles (Fig. 2(f)).

Owing to the mesoporous silica matrix, the Au and Ag nanoparticles@mesoporous silica samples demonstrate type-IV $\mathrm{N}_{2}$ adsorption-desorption isotherms. From these isotherms, capillary condensation occurs at $P / P_{0}$ ratios ranging from 0.6 to 0.9 (Fig. 3(a)). After estimation by the BET method and BJH calculation, both of the samples showed BET surface areas larger than $300 \mathrm{~m}^{2} \mathrm{~g}^{-1}$, and pore size distributions centered at $6.0-8.0 \mathrm{~nm}$. According to these results, the Au and Ag nanoparticles with a large
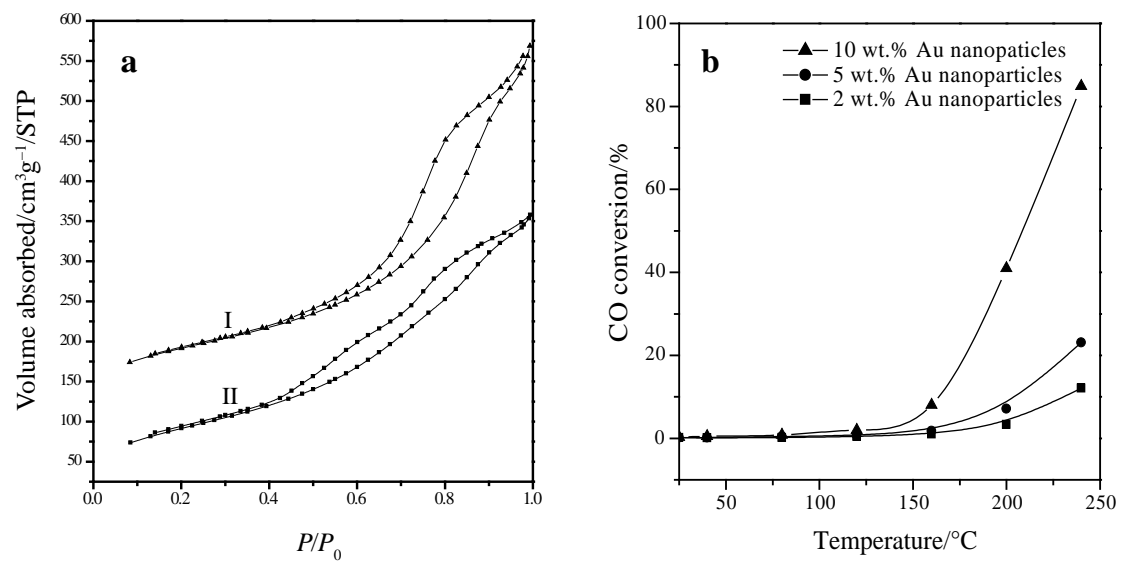

Fig. 3. (a) $\mathrm{N}_{2}$ adsorption-desorption isotherms of calcined Au and Ag nanoparticles@ mesoporous silica (curve I: Au nanoparticles; curve II: Ag nanoparticles); (b) CO conversion of $\mathrm{Au}$ nanoparticles@mesoporous silica at different reaction temperatures. 
surface area and a large pore size can be conveniently synthesized by our method. This synthetic method can be extensively used to prepare other Ag-Au alloy nanoparticles@ mesoporous silicas. The TEM image also reveals the excellent dispersion of the Ag-Au alloy nanoparticles within the mesoporous silica.

In practice, because the Au nanoparticles were well dispersed within the small mesoporous silica particles (i.e., less than $200 \mathrm{~nm}$ ), the Au nanoparticles are accessible to the environment. Thus, the Au nanoparticles@mesoporous silica can be used as a highefficiency catalyst, particularly for CO oxidation. ${ }^{(13,14)}$ The resulting Au nanoparticles@ mesoporous silica showed catalytic capability of CO oxidation. CO conversion rate increases with increasing reaction temperature and $\mathrm{Au}$ nanoparticle content (Fig. 3(b)). When $\mathrm{Au}$ nanoparticle content increases to $10 \mathrm{wt} . \%$, the $\mathrm{CO}$ conversion can reach approximately $90 \%$ at $240^{\circ} \mathrm{C}$. Because the catalytic capability is insufficient at low temperatures, other experimental factors, including hydrothermal conditions and the chemical compositions of the noble metal nanoparticles, will be further studied to generate a high-performance catalyst for $\mathrm{CO}$ oxidation or other large-molecule reactions.

In addition to the application of Au nanoparticles@mesoporous silica to catalytic reaction, bare Au nanoparticles on the surface of silica particles could have direct contact with the absorbed molecules or organics on the Au nanoparticles@mesoporous silica. In addition, the silanol groups (-Si-OH) of the silica matrix have a high absorption affinity with the bioorganics via hydrogen bonding interactions.(3) Thus, the Au nanoparticles@ mesoporous silica could be considered for use in SERS applications. Figure 4 shows the Raman and SERS spectra of S. aureus and nanoparticles. Figure 4(a) shows a normal Raman spectrum of $S$. aureus under 10× PBS solution with a concentration of

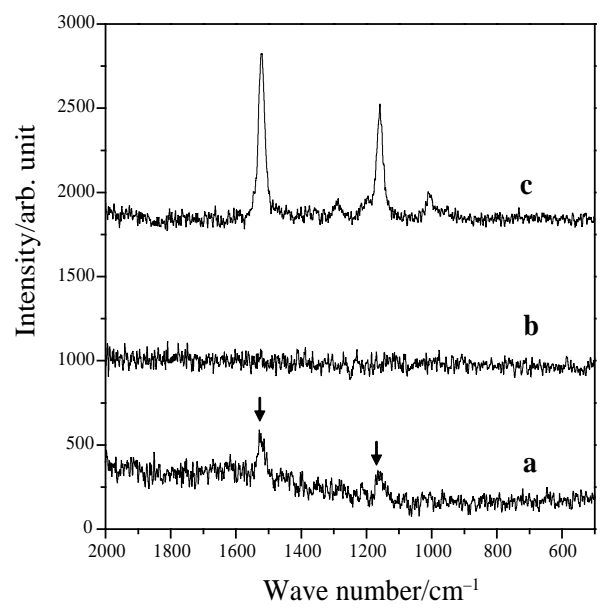

Fig. 4. The Raman and SERS spectra of S. aureus and Au nanoparticles@mesoporous silica. (a) Raman spectrum of S. aureus (10× PBS, pH 7.4); (b) the Raman spectrum of Au nanoparticles@ mesoporous silica; (c) SERS spectrum of S. aureus attached Au nanoparticles@mesoporous silica. Radiation of $633 \mathrm{~nm}$ was used for excitation. 
$10^{9} / \mathrm{ml}$ (pH 7.4). The signal indicated by arrows comes from $\beta$-carotene in S. aureus. The Raman spectrum of $S$. aureus is predominated by the strong bands centered at 1155 and $1523 \mathrm{~cm}^{-1}$, assigned as the $\mathrm{C}-\mathrm{C}$ stretch vibration and $\mathrm{C}=\mathrm{C}$ stretch vibration for $\beta$-carotene. ${ }^{(10)}$ Figure 4(b) shows the Raman spectra of the Au nanoparticles@mesoporous silicas. The inorganic Au nanoparticles@mesoporous silicas show no vibrational bands within this range for detecting the organics. Thus, the Au nanoparticles@mesoporous silicas could be a superior material for SERS detection. As can be seen in Fig. 4(c), the SERS spectrum of $S$. aureus exhibited some peak shifts compared with the Raman spectrum of S. aureus, e.g., 1154 to $1158 \mathrm{~cm}^{-1}$ and 1523 to $1529 \mathrm{~cm}^{-1}$. These changes may be due to interactions with nanoparticles and/or neighboring molecules. ${ }^{(11)}$ A comparison of Figs. 3(a) and 3(c) indicates that the SERS spectrum of $S$. aureus shows a strong enhancement (about one-order enhancement) of its characteristic bands (i.e., peak at approximately $1529 \mathrm{~cm}^{-1}$ ). According to a previous report, ${ }^{(11)}$ these changes may be attributed to the direct attachment between organic molecules and the Au nanoparticle surface, and therefore to changes in the SERS effect (electromagnetic or chemical enhancement). ${ }^{(12)}$ The enhancement efficiency of the Au nanoparticles@mesoporous silica on the Raman spectrum is evident but not as large as that of the large Au nanoparticles $(\approx 50 \mathrm{~nm})$. This is because the particle size of the Au nanoparticles in Au nanoparticles@mesoporous silicas is extremely small and such silicas have a low Au nanoparticle content $(\approx 4.0 \mathrm{wt} . \%)$. The enhancement effect could be markedly improved by increasing the size and content of the Au nanoparticles in the mesoporous silicas.

\section{Conclusion}

In conclusion, we provided a new and convenient one-pot method to directly synthesize a large-surface-area noble nanoparticles@mesoporous silica material that can be used in SERS and CO oxidation applications. In addition, the mesoporous silica has many silanol groups (Si-OH) on the surface, which can be easily modified with silanes of different functional groups via a simple reflux procedure. With appropriate selection of such silanes, the surface properties of metal nanoparticles@mesoporous silicas could be tuned to have a high affinity to the analyzed biomolecules and reactants via matching interactions.

\section{Acknowledgements}

The research was financially supported by the National Science Council of Taiwan (NSC95-2113-M-006-011-MY3 and NSC95-2120-M-006-009) and Landmark Project, National Cheng Kung University.

\section{References}

1 C. T. Kresge, M. E. Leonowicz, W. J. Roth, J. C. Vartuli and J. S. Beck: Nature 359 (1992) 710 .

2 D. Zhao, J. Feng, Q. Huo, N. Melosh, G. H. Fredrickson, B. F. Chmelka and G. D. Stucky: Science 279 (1998) 548. 
3 J. Y. Ying, C. P. Mehnert and M. S. Wong: Angew. Chem., Int. Ed. 38 (1999) 57.

4 H. P. Lin and C. Y. Mou: Acc. Chem. Res. 35 (2002) 927.

5 R. K. Iler: The Chemistry of Silica: Solubility, Polymerization, Colloid and Surface Properties, and Biochemistry (Wiley, New York, 1979).

6 Y. C. Lin, C. H. Hsu, H. P. Lin, C. Y. Tang and C. Y. Lin: Chem. Lett. 36 (2007) 1258.

7 R. Brayner, T. Coradin, M.-J. Vaulay, C. Mangeney, J. Livage and F. Fiévet: Colloids Surf., A 256 (2005) 191.

8 M. Haruta: Chem. Rec. 3 (2003) 75.

9 G. C. Bond and D. T. Thompson: Catal. Rev. -Sci. Eng. 41 (1999) 319.

10 P. Rösch, M. Schmit, W. Kiefer and J. Popp: J. Mol. Struct. 661-662 (2003) 363.

11 C. A. Murray and S. Bodoff: Phys. Rev. Lett. 52 (1984) 2273.

12 D. S. Grubisha, R. J. Lipert, H. Y. Park, J. Driskell and M. D. Porter: Anal. Chem. 75 (2003) 5936.

13 J. H. Liu, A. Q. Wang, Y. S. Chi, H. P. Lin and C. Y. Mou: J. Phys. Chem. B 109 (2005) 40.

14 Z. Wang, A. Bonoiu, M. Samoc, Y. Cui and P. N. Prasad: Biosens. Bioelectron 23 (2008) 886. 